\title{
Fiber Optic Sensing Systems Using High Frequency Resonant Sensing Heads With Intensity Sensors
}

Grigory Adamovsky

Lewis Research Center

Cleveland, Ohio

and

Duncan J. Maitland IV

Cleveland State University

Cleveland. Ohio

(AASE-TU-1C1318) FIEEE CETIC SEASING

SYSIENS CSING EIGE FBECUENCY EESCAANT

SEASING EEADS FIIB INTENSIIY SFNSCRS (NASA)

C F CSCL 14B

Unclas

N88-28293

G3/35 C158988

Prepared for the

High Bandwidth Analog Applications of Photonics II Conference sponsored by the Society of Photo-Optical Instrumentation Engineers Boston, Massachusetts, September 8-9, 1988 
Fiber optic sensing systems:using high frequency resonant selsing heads with. intensity sensors:

\title{
Grigory Adamovsky
}

National Aeronautics and Space Administration, Lowis Research Center cleveland, Ohio 44135

\author{
and \\ Duncan J. Maitland IV \\ Cleveland state University, Department of Engineering Technology \\ Cleveland, Ohio 44115
}

\section{: ABS'IRACT}

Optical fibers have an inherent capability of transmitting high bandwidth analog and digiin tal signals. To apply this property of fiber optics to remote sensing, special sensing . heads as well as signal processing electronics have to be developed. In systems employing

w intensity modulating sensors, there is also a need for a referencing technique to compensate for changes in the transmission of the connecting fibers and light source intensity.

This paper discusses fiber optic sensing systems using intensity sensors incorporated in sensing heads of a special configuration. Different modes of operation as well as resonant conditions are explained. Theoretical and experimental analyses are also given.

\section{INTRODUCTION}

Fiber-optic sensing systems have found wide applications in various fields of industry due to EMP immunity, low weight and volume, and broad bandwidth capabilities. In these systems a sensor incorporated in a sensing head responds to changes in a measured parameter by modulating the light passing through it. The sensor is an element that is being affected by the measured parameter and reacts to the changes in this parameter by encoding or modulating an optical signal. The measured parameter acting on the sensor could modulate intensity or phase of this signal, affect the time constant, optical frequency, and other parameters of the initial optical signal. I The sensing head also plays an important role in the way the system operates. The purpose of the sensing head is to. support the modulation or encoding of the optical signal. The sensing head provides, if needed, referencing, compensation, delay, and other features that promote the given mode of operation and sometimes determine the type of sensing system. ${ }^{2}$

Resonators form a special group of $f$ iber-optic sensing heads that respond selectively to specific frequencies of a signal passing through them. A simple example of a resonator is an optical interferometer. However, their use at optical frequencies has several disadvantages. Fiber optic interferometers are phase sensitive devices with complex stabilizing and signal processing electronics. Also, they must employ single-mode fibers in order to achieve the maximum efficiency of the sensing system. On the other hand, sensors that modulate the intensity of the light are attractive because of their.simplicity and low cost.

This paper describes and analyzes the performance of fiber optic sensing systems with intensity modulating sensors incorporated in resonant sensing heads.

\section{SYSTEM ANALYSIS}

Schematics of different interferometric sensing heads are presented in Fig. 1 . A common factor among interferometric heads is that they split the initial optical signal into two or more signals, delay one of these signals with respect to the others, and then recombine them producing a resultant signal. Resonant conditions occur when the initial signal contains frequency components with periods that are equal to or are an integral multiple of the time delay.

For theoretical and experimental analyses, a reflective Fabry-Perot type interferometric sensing head has been chosen. Schematics of such a sensing head have already been reported. The sensing head consists of a piece of multimode fiber placed between two mirrors. One of the mirrors is a semitransparent, semireflective one, and the other is a reflector. An intensity sensor is incorporated in the interferometric sensing head and can be either intrinsic or extrinsic. The experimental setup is shown in Fig.. 2 . It consists of a light source with a driver-modulator, a sensing head with an intensity sensor, a photodetector 
(PD) with signal processing electronics; and optical fibers connecting them. A laser diode (LD) is used as a light source. Two mirrors, Ml and M2, with a $1-m$ long fiber L placed between them form a sensing head. The reflection and transmission coefficients of mirror Ml control the amount of optical power launched into the sensing head. The mirror-reflector M2 is positioned on a translation stage. so a distance between the fiber end and the reflective surface of the mirror could be varied. This provides an intensity modulation of the optical signal passing through the sensing head.

The time varying optical signal emitted by the source experiences multiple reflections in the sensing head. Thus, the resultant signal $x(t)$ could be represented as a sum of $t i m e-$ delayed signals with the first of these signals being due to the first reflection from the semireflecting mirror Ml:

$$
x(t)=\sum_{m=1}^{\infty} a_{m} x[t-(m-1) \Delta],
$$

where $m$ is a positive integer; a is a coefficient determined by the number m, as well as reflection and transmission coefficients of the mirrors and total losses in the sensing head; $\Delta$ is a time delay between the signals exiting the sensing head.

The time delay $\Delta$ in the system used is:

$$
\Delta=\frac{2 n L}{C} \text {. }
$$

where $c$ is the speed of light in a vacuum and $n$ is the group index of refraction of the fiber in the sensing head.

If the Fourier transform of the first signal $a_{1} x(t)$ is $F_{1}(j w)$, then the Fourier transform of the entire resultant signal would be:

$$
F(j w)=F_{1}(j w)\left\{\sum_{m=1}^{\infty} \frac{a_{m}}{a_{1}} \exp [-j(m-1) w \Delta]\right\} .
$$

If the mirrors used have such reflection and transmission coefficients that the secondary reflections are insignificant, then the number of exiting optical signals would be limited to two $(m=2)$, and Eq. (3) could be simplified:

$$
F(j w)=F_{1}(j w)\left[1+\frac{a_{2}}{a_{1}} \exp (-j w \Delta)\right] \text {. }
$$

The last equation can be rewritten in a phasor form:

$$
F(j w)=\left|F_{1}(j w)\right| \exp (j \psi)\left[1+\alpha^{2}+2 \alpha \cos (w \Delta)\right]^{0.5} \exp (j \phi) \text {. }
$$

where the first two terms $\left|F_{1}(j w)\right| \exp (j \psi)$ are a phasor representation of a Fourier transform $F_{1}(j w), \alpha=a_{2} / a_{1}$, and

$$
\phi=\arctan \left[\frac{-\alpha \sin (\omega \Delta)}{1+\alpha \cos (w \Delta)}\right] \text {. }
$$

A plot representing the coefficient $\left[1+\alpha^{2}+2 \alpha \cos (w \Delta)\right]^{0.5}$ as a function of argument wD for different values of $a$ is shown in Fig. 3 .

In the following sections we will discuss systems of the same configuration as presented above but using two different driving conditions for the light source, high speed pulsing and $C W$ modulation. The results in both cases will be described by applying the theory introduced in this section.

\section{PULSE MODULATION}

In a system with a pulsed modulated light source, the sensing head generates secondary pulses whose amplitudes encode information about the sensed parameter (in this case a displacement). Such a system has already been described and a signal processing technique to analyze the resultant signal has been proposed.3,4. Assume that the signal emitted by the 
source is a periodic rectangular light pulse with a period. $T$ and a duration ti. Then, following the previous analysis, the amplitude spectrum of the resultant signal detected could be written as:

$$
F\left(w_{m}, \alpha\right)=\frac{A_{1} T_{1}}{T}\left|\frac{\sin \left(w_{m} t_{1} / 2\right)}{w_{m} t_{1} / 2}\right|\left[1+\alpha^{2}+2 \alpha \cos \left(w_{m} \Delta\right)\right]^{0.5},
$$

where the amplitudes of the primary. and secondary pulses are given by $A_{1}$ and $\alpha A_{1}$, respectively, and $w_{m}=2 \pi m / T .$. In case the delay $\Delta=T / 2$ then

$$
F\left(w_{m}, \alpha\right)=\frac{A_{1}}{m \pi}\left|\sin \left(m \pi t_{1} / T\right)\right|\left[1+\alpha_{i}^{2}+2 \alpha \cos (m \pi)\right]^{0.5} \text {. }
$$

A signal processing scheme for analyzing the resultant signal has already been described. 5 It is based on splitting the generated electric signal into two branches (low frequency and high frequency), filtering the appropriate frequency components, processing these components separately, and taking a ratio of the resultant signals. After the signal is split and passed through bandpass filters centered at the first ( $m=1$ ) and the second (m=2) harmonics. the ratio of amplitudes of these harmonics is:

$$
R=\frac{F\left(w_{1}, \alpha\right)}{F\left(w_{2}, \alpha\right)}=\frac{1}{\cos \left(\pi t_{1} / T\right)} \frac{|1-\alpha|}{1+\alpha}
$$

The experimental data for this case has been presented in Ref. 5 .

For $T=4 t_{1}$. Eq. (10) can be simplified:

$$
R=(2)^{0.5} \frac{|1-\alpha|}{1+\alpha} \text {. }
$$

Despite the fact that the technique works at any value of $T, t_{1}$, and $\Delta$, the system performs best when the repetition rate $T$ and the length $L$ of the fiber in the sensing head are related as:

$$
\mathrm{T}=4 \mathrm{knL} / \mathrm{c} \text {. }
$$

where $k$ is an integer and $T=2 \Delta$.

A similar resonant condition occurs when the initial signal sent into the sensing head is in the form of a double pulse, and the delay $\delta$ between pulses in the initial signal is equal to $2 \Delta$. Assuming that the amplitudes of the pulses in the initial double pulse are the same, the amplitude spectrum of the resultant signal could be written as:

$$
F\left(w_{m}, \alpha\right)=\frac{2 A_{1} t_{1}}{T}\left|\operatorname{sinc}\left(m t_{1} / T\right) \cos \left(w_{m} \Delta\right)\right|\left[1+\alpha^{2}+2 \alpha \cos \left(w_{m} \Delta\right)\right]^{0.5} .
$$

The phenomenon that occurs when an. initial double pulse is used, has been already observed and the results of the observations have been presented in Ref. 5 . During the experiment, frequency bands have been clearly identified. The behavior of these frequency bands is similar to that of separate frequencies for the case of a symmetric pulse. The presence of frequency bands is attributed to the initial double pulse repetition rate. The pulse duration $t_{1}$ is 5 nsec and the repetition rate of the initial signal is about $10 \mathrm{kHz}$. The delays are chosen to be $\delta=20$ nsec and $\Delta=10$ nsec. The bandpass filters used to separate the frequency bands are centered at frequencies $50 \cdot \mathrm{MHz}$ and $100 \mathrm{MHz}$ and have half-power bandwidths of about $13 \mathrm{MHz}$ and $25 \mathrm{MHz}$, respectively. Experimental data for this case obtaining a range of displacements from 0 to $600 \mu \mathrm{m}$ is shown in Fig. 4.

In order to process the resultant signal, both techniques require filtering and separate processing of either two frequency components or frequency bands of the signal. Although the processing schemes are. completely analogous, the bandwidth of the filters is critical. Any shift in frequency or change in the filter width can cause a loss of the outer frequency bands, reducing the system's sensitivity.

\section{CONTINUOUS MODULATION}

In the case of a continuous sinusoidal modulation of the light source intensity at RF $f r e-$ quencies, the sensing head described performs as an interferometer whose output is the superposition of two sinusoidal waves with their phase difference dependent on the cavity delay. Note that the coherence length of the light source is much shorter than the cavity length so 
no optical interference effects are observed.: Thus, the theory developed and Eqs. (1) to. (6) can be applied. The Fourier spectrum of the resultant signal in this cașe is:

$$
F(j w)=A_{1}\left[1+\alpha^{2}+2 \alpha \cos (w \Delta)\right]^{0.5} \exp (-j w \phi) \text {. }
$$

where $w$ is angular modulation frequency, $A_{1}$ is the amplitude of the primary" signal which is generated by a portion of the initial signal reflected from mirror Ml back to the detector. (See Figure 2).

It is clear that a sensing system using continuous modulation in the present configuration does not have a reference channel to compensate for variable losses in the system. The reference channel has been constructed by using two modulating frequencies. To obtain a resonant condition, the frequencies $f_{1}$ and $f_{2}$ have been chosen so that $f_{2}=2 f_{1}$ and $f_{2}=c / 2 n L$. These two frequencies correspond to destructive and constructive interference in the sensing head with a fiber $L$ meters long. Thus, the ratio of the magnitude of the resultant signal obtained at low frequency $f_{1}$ to that obtained at high frequency $f_{2}$. $\mathrm{V}_{\mathrm{LF}} / \mathrm{V}_{\mathrm{HF}}$, is:

$$
R=\frac{A_{1}}{B_{1}} \frac{|1-\alpha|}{1+\alpha} \text {, }
$$

where $A_{1}$ is the amplitude of the signal described by Eq. (13) with the modulating frequency $f_{l}$ of the initial signal corresponding to destructive interference in the sensing head, $B_{1}$ is the amplitude of the signal described by Eq. (13) with $A_{1}$ replaced by $B_{1}$ and the modulating frequency $\mathrm{f}_{2}=2 \mathrm{f}_{1}$ of the initial signal corresponding to constructive interference in the sensing head. The amplitudes $A_{1}$ and $B_{1}$ remain in the $f$ inal expression for $V_{L F} / V_{H F}$. This means that the sensitivity of the system could be altered by intentionally changing one of the RF-driving signals with respect to the other.

Equations (10) and (14) are very similar and if the amplitudes of the initial modulated signals are equal, then Eq. (14) can be simplified to:

$$
R=\frac{|1-\alpha|}{1+\alpha}
$$

During the experiment, a laser was driven at modulating frequencies of $50 \mathrm{MHz}$ and $100 \mathrm{MHz}$ in order to obtain destructive and constructive interference, respectively, in the sensing head with a $1-m$ long fiber. The resultant signals at these frequencies were ratioed, and according to Eq. (14) the ratio was independent of intensity losses. To verify this, the intensities of modulated signals were decreased to 75 percent of their initial values. The data for both cases with a range of displacements from 0 to $600 \mu m$ are shown in Fig. 5 . The maximum deviation of the ratio $V_{L F} / V_{H F}$ does not exceed 1.5 percent of the initial value at the corresponding data point. During the experiment, a resolution of less than 1 m was observed.

\section{CONCLUSION}

It has been shown in this paper that the configuration of a sensing head plays an important role in the system's performance. Sensing heads configured as interferometers coupled with appropriate source modulation techniques provide good referencing. and high resolution. The demonstrated insensitivity of the system to intensity losses makes it useful for sensing in a harsh environment. Also, the use of high frequencies for generating and analyzing data broadens the bandwidth of the system. At the same time, the advantage of fiber optics very broad bandwidth capability is utilized more effectively.

\section{ACKNOWLEDGMENT}

One of the authors (D.J. Maitland) wishes to acknowledge the support of the NASA Lewis Research Center (Grant NCC-3-58).

\section{REFERENCES}

1. B. Culshaw, Optical Fibre Sensing and Signal Processing, Peter Peregrinus, London, (1984).

2. G. Adamovsky, "Referencing in Fiber Optic: Sensing systems," in Optical Techniques for Sensing and Measurement in Hostile Environments. C.H. Gillespie and R.A. Greenwell, eds., Proc. SPIE 787, 17-23 (1987). 
3. G. Adamovsky. "Amplitude Spectrum Modulation Technique for Analog Data Processing in Fiber Optic Sensing System with Temporal Separation of Channels," in Fiber Optin and Laser Sensors V. R.P. DePaula and E. Udd, eds., Proc. SPIE 838, 264-270 (1987).

4. G. Adamovsky, "Fiber-Optic Displacement Sensor with Temporally Separated Signal and Reference Channels," Appl. Opt. 27(7) 1313-1315 (1988).

5. G. Adamovsky and D.J. Maitland IV, "Fiber Optic Sensors with Internal Referencing," to be published in Optical Sensing and Metrology II, C.P. Grover, ed., Proc. SPIE 954 $(1988)$.

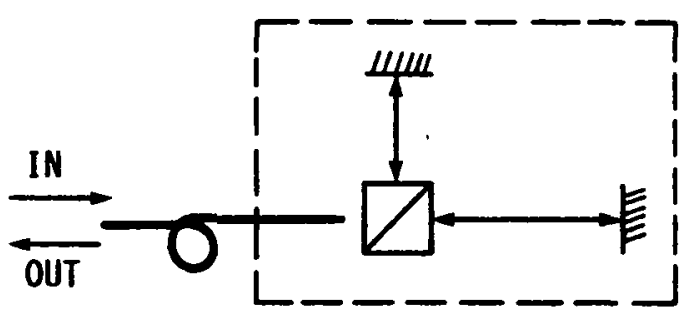

MICHELSON INTERFEROMETER WITH BEAM SPLITTING CUBE

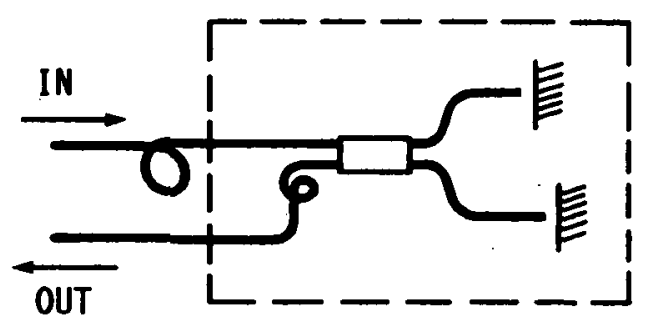

MICHELSON INTERFEROMETER

WITH $2 \times 2$ FIBER OPTIC COUPLER/SPLITTER

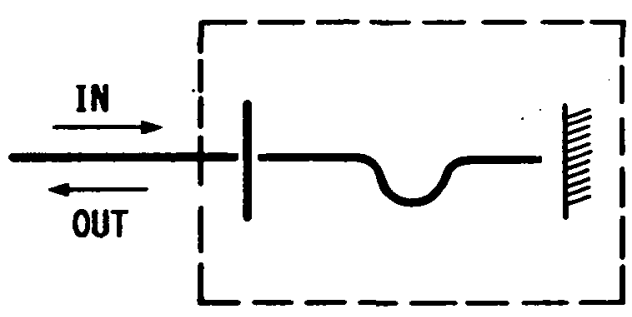

FABRY-PEROT INTERFEROMETER IN REFLECTION MODE

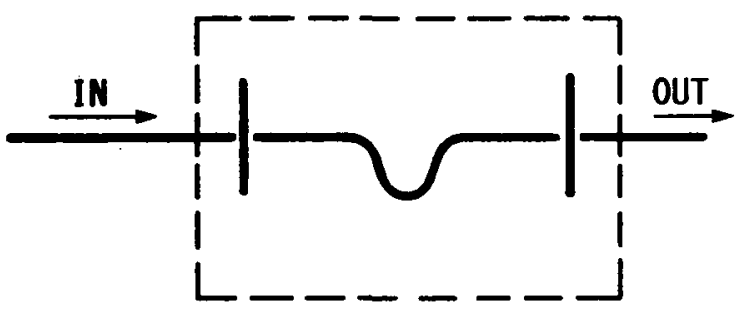

FABRY-PEROT INTERFEROMETER IN TRANSMISSION MODE

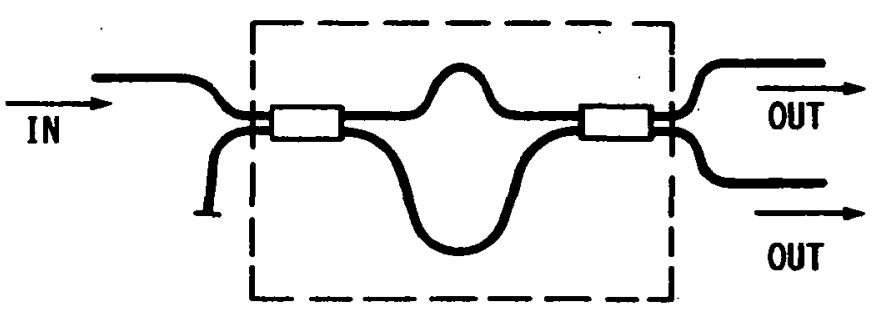

MACH-ZEHNDER INTERFEROMETER

IN TRANSMISSION MODE

FIGURE 1. SCHEMATICS OF FIBER OPTIC INTERFEROMETRIC HEADS. 


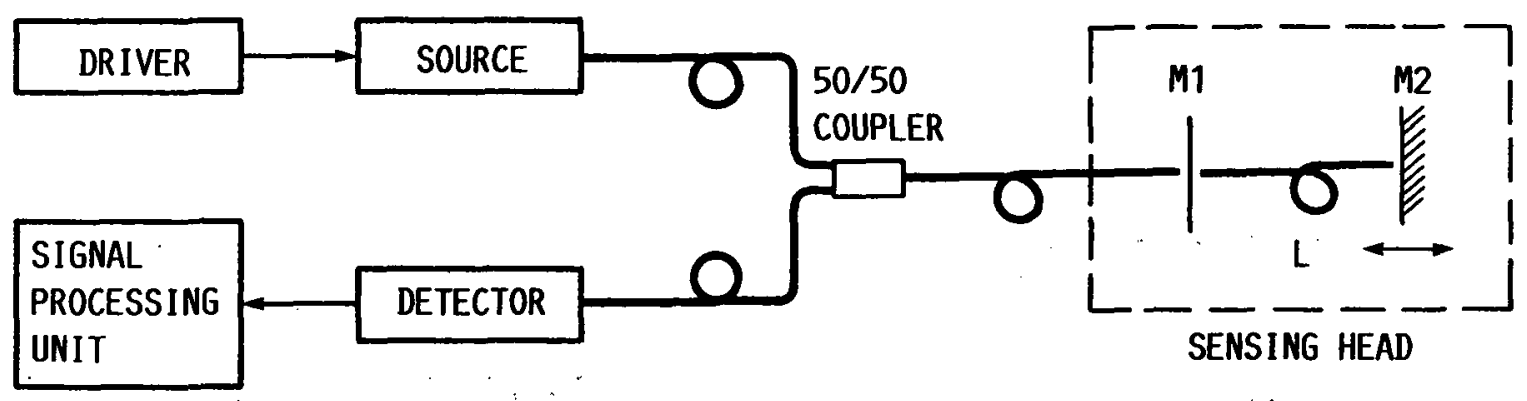

FIGURE 2. EXPERIMENTAL SETUP.

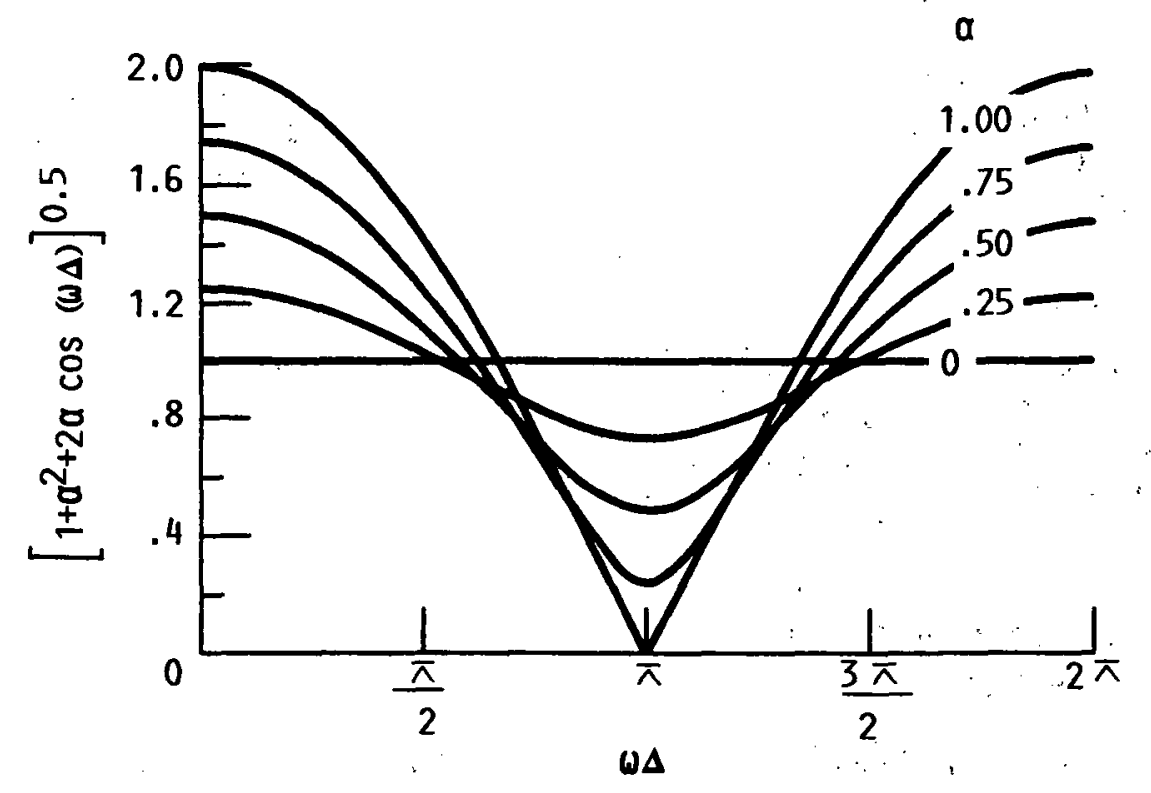

FIGURE 3. COEFFICIENT $\left[1+\alpha^{2}+2 \alpha \cos (\omega \Delta)\right] 0.5$ AS A FUNCTION OF ARGUMENT WA FOR DIFFERENT VALUES OF $\boldsymbol{\alpha}$. 


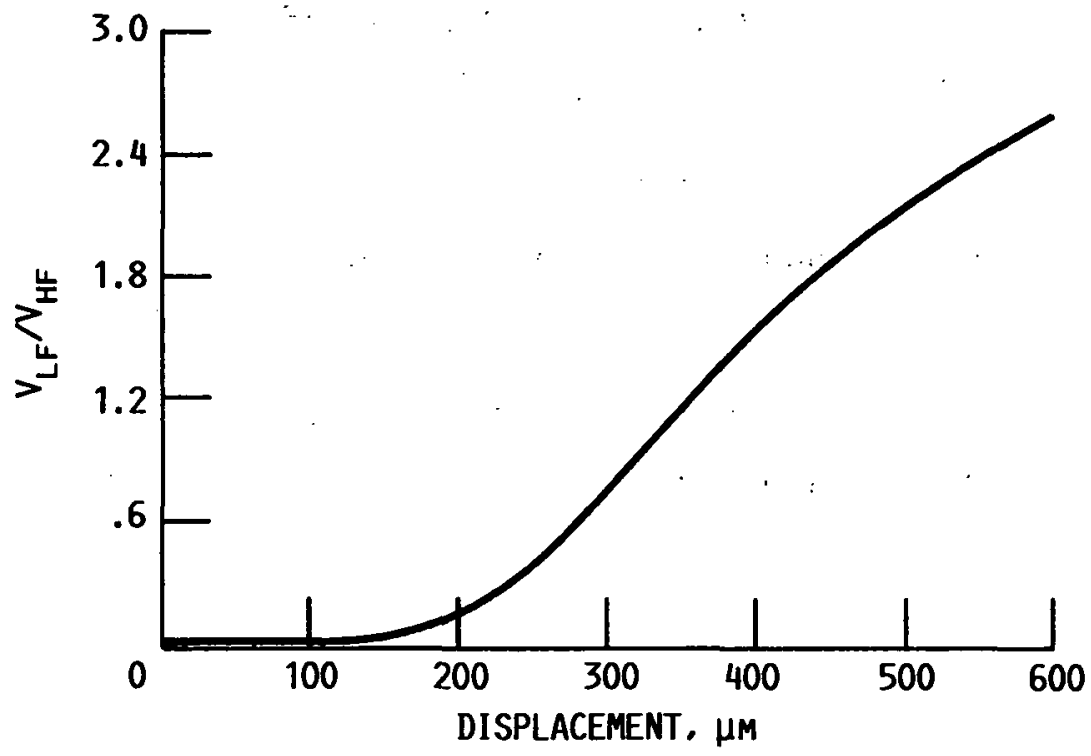

FIGURE 4. $\quad V_{L F} N_{\text {HF }}$ AS A FUNCTION OF DISPLACEMENT IN A SYSTEM WITH INITIAL DOUBLE PULSE.

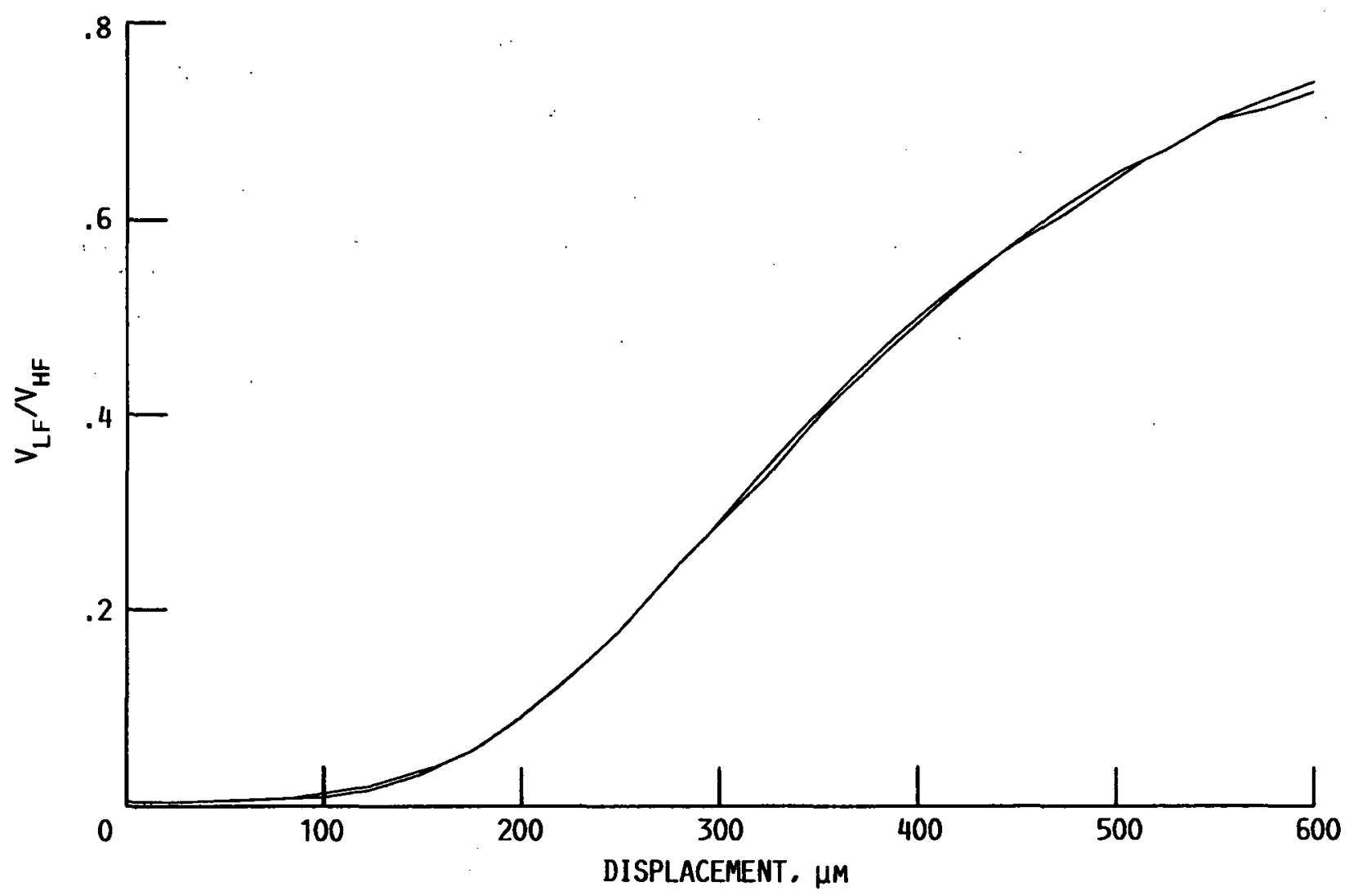

FIGURE 5. $\quad V_{L F} N_{H F}$ AS FUNCTION OF DISPLACEMENT IN SYSTEM USING TWO MODULATING RF FREQUENCIES. ONE OF THE CURVES REPRESENTS A CASE WHEN INTENSITIES OF MODULATED SIGNALS ARE DECREASED TO 75 PERCENT OF THEIR INITIAL VALUES. 


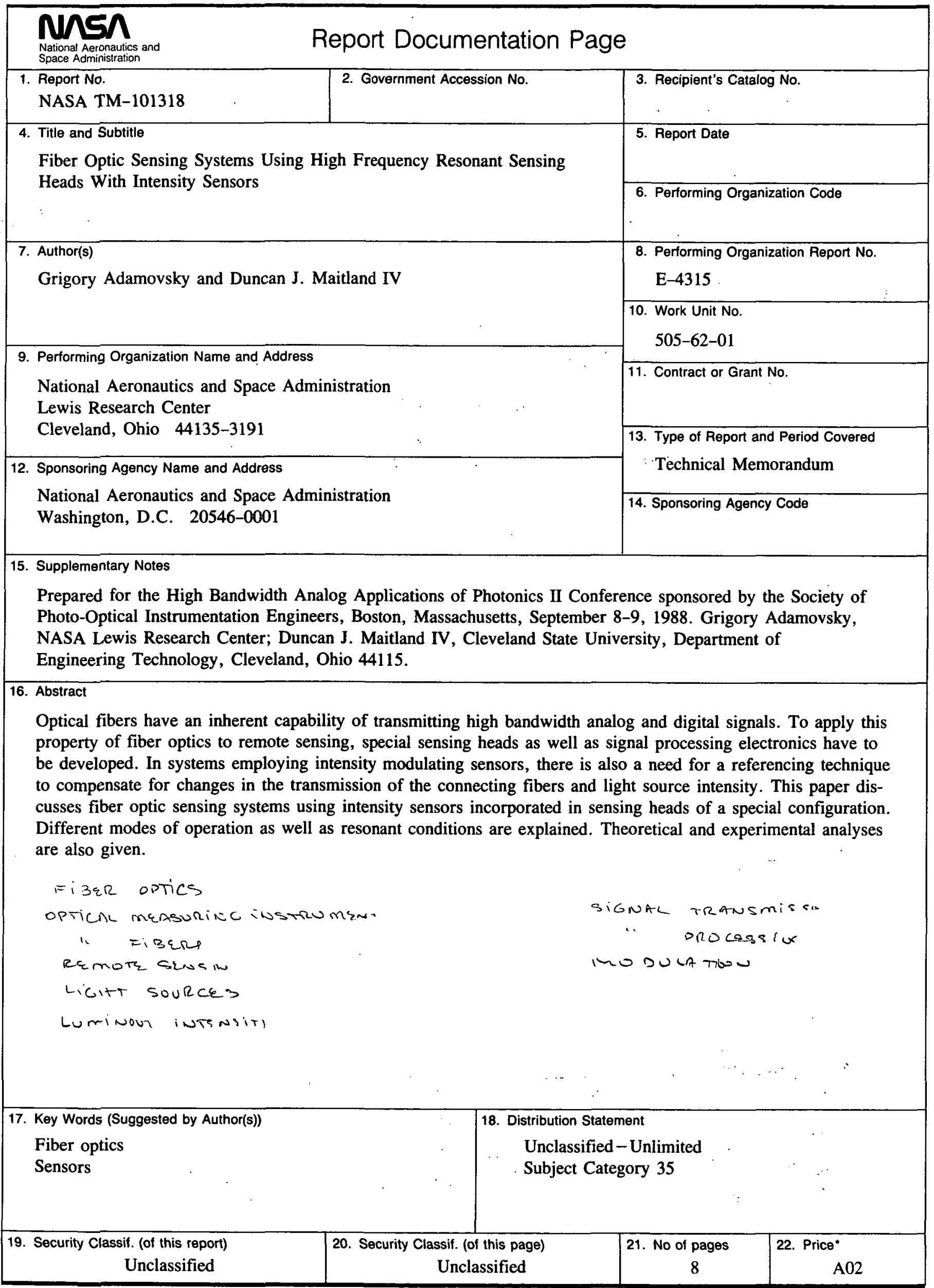


National Aeronautics and Space Administration

Lewis Research Center

Cleveland, Ohio 44135

Officia! Business

Penalty for Private Use $\mathbf{5 3 0 0}$

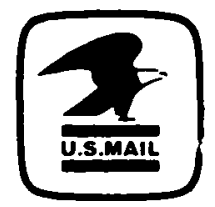

Postage and Fees Paid

National Aeronautıcs and

Space Admınıstratıon

NASA 451 